\title{
MM-Based Solution for Partially Connected Hybrid Transceivers with Large Scale Antenna Arrays
}

\author{
Aakash Arora, Christos G. Tsinos, Bhavani Shankar Mysore R, Symeon Chatzinotas and Björn Ottersten \\ Interdisciplinary Centre for Security, Reliability and Trust (SnT), University of Luxembourg \\ email address: \{aakash.arora, christos.tsinos, bhavani.shankar, symeon.chatzinotas, bjorn.ottersten\}@uni.lu
}

\begin{abstract}
In a mmWave multiple-input multiple-output (MIMO) communication system employing a large-scale antenna array (LSAA), the hybrid transceivers are used to reduce the power consumption and the hardware cost. In a hybrid analog-digital (A/D) transceiver, the pre/post-processing operation splits into a lower-dimensional baseband (BB) pre/postcoder, followed by a network of analog phase shifters. Primarily two kinds of hybrid architectures are proposed in the literature to implement hybrid transceivers namely, the fully-connected and the partially-connected. Implementation of fully-connected architecture has higher hardware complexity, cost and power consumption in comparison with partially-connected. In this paper, we focus on partially-connected hybrid architecture and develop a low-complexity algorithm for transceiver design for a single user point-to-point mmWave MIMO system. The proposed algorithm utilizes the variable elimination (projection) and the minorization-maximization (MM) frameworks and has convergence guarantees to a stationary point. Simulation results demonstrate that the proposed algorithm is easily scalable for LSAA systems and achieves significantly improved performance in terms of the spectral efficiency (SE) of the system compared to the state-of-the-art solution.

Index Terms-Hybrid precoding, minorization-maximization, MM, mmWave MIMO communications, large-scale antenna arrays.
\end{abstract}

\section{INTRODUCTION}

Emerging wireless networks demand several orders of increase in capacity to deliver higher data-rates. To achieve this a large-scale antenna array (LSAA) multiple-input multipleoutput (MIMO) communication system is generally employed, which uses a large number of transmitting and receiving antennas. The use of LSAA is an essential requirement in mmWave bands, has gained attention recently because of the availability of unused spectrum. The communication at mmWave frequencies generally suffers from several losses including those due to propagation, penetration loss, etc., [1][5]. Thus, LSAAs are generally employed at the transceivers to achieve high beamforming gains and to mitigate the losses associated with the system. However, from an implementation point of view, each transmitting and receiving antenna has to be driven by a radio frequency (RF) chain. Thus, the number of $\mathrm{RF}$ chains increase linearly with the number of antennas. This requirement becomes the bottleneck in the implementation of the system because it substantially increases the power consumption and hardware cost.

This work is supported by the National Research Fund (FNR), Luxembourg under the AFR-PPP grant for Ph.D. project SPASAT (Ref.: 11607283), the CORE-PPP project PROSAT, ECLECTIC, and CI-PHY.
Several precoding/postcoding solutions have been proposed in the literature to alleviate the aforementioned issues. In [6][8], the analog beamforming solution was considered, where a network of analog phase-shifters is driven using only one RF chain. But this architecture is not suitable for transmission of multiple streams. Another alternative uses analog switches [9]-[11], which unfortunately degrades the array gain. In order to achieve high beamforming gain without compromising the ability to transmit multiple streams hybrid analog-digital (A/D) transceivers were (first) proposed in [1]. In this architecture, the pre/postcoding operation splits into a smaller dimensional digital baseband (BB) pre/postcoder followed by a network of analog phase-shifters.

For a mmWave communication system, hybrid transceivers were (first) proposed in [2]. It is shown that the near-optimal hybrid transceiver is obtained by maximizing the SE of the system. As the SE maximization jointly over the hybrid precoder and postcoder is difficult, the problem is further decoupled into precoder and postcoder optimization. It is also shown that the hybrid precoder design problem is approximated by minimizing the Frobenius norm of the difference between the fully digital and the product of analog-digital precoders [3]. It is important to note that the problem of designing the hybrid precoder and postcoder has a similar mathematical structure. This enables the reuse of algorithms developed for the transmitter to implement the postcoder as well. This motivates the focus on a hybrid precoder design problem, and apply the same algorithms at the receiver for performance evaluation.

Apart from the aforementioned works, a number of works exist which deal with the hybrid design problem [5], [12][21]. These works are categorized as codebook based and codebook free. In codebook based methods, the analog and digital precoders are pre-computed and stored in a codebook structure; this facilitates the limited channel feedback from the user to the base station. In [3], the analog precoder is predetermined using a codebook and the resulting optimization problem is solved using sparse reconstruction techniques [22]. Instead of using predetermined codebooks, in codebook free approaches, the Frobenius norm approximation problem is directly solved using alternating minimization algorithms [12].

Among the different architectures for hybrid transceiver design, the two most common ones are the fully-connected and the partially-connected [23]. In the fully-connected case, each $\mathrm{RF}$ chain is connected to all the transmitting antennas, whereas 
in the partially-connected case only a subset of antennas is connected to an RF chain. Although the partially-connected architecture has slightly reduced performance compared to the fully-connected [5], [23], it exhibits lesser hardware complexity, cost, and power consumption [5], which makes it a more attractive candidate for a practical implementation of the system. In [12], SDR-ALTMIN algorithm is presented for the partially-connected case, which uses alternating minimization and requires external toolboxes to solve the problem. Additionally, there are no convergence guarantees and it has high computational complexity.

The aim of this paper is to provide a low-complexity solution with convergence guarantees. We follow the codebook free approach and directly address the Frobenius norm approximation criterion to design the hybrid transceiver. The approximated problem can also be viewed as a constant modulus constrained matrix factorization (CMCMF) problem. We briefly mention the following contributions:

- A novel algorithm for the partially-connected hybrid transceiver architecture using the combination of variable elimination and the minorization-maximization (MM) framework.

- The proposed algorithm has convergence guarantees, lower complexity, and improved performance compared to the state-of-the-art solution as illustrated in Section IV. This augurs well with the low-complexity, powerefficient, partially-connected architecture.

\section{System Model and Problem Formulation}

We first describe a point-to-point single user mmWave MIMO system employing hybrid pre/post-coding and provide necessary input-output models. Subsequently, we formulate the precoder optimization problem.

\section{A. System Model}

We consider a system equipped with $T_{x}$ transmitting and $R_{x}$ receiving antennas. The transmitter has $N_{t}<<T_{x} \mathrm{RF}$ chains whereas receiver has $N_{r}<<R_{x}$ RF chains. The system is designed to communicate $N_{s}$ data streams, where $N_{s} \leq \min \left\{N_{t}, N_{r}\right\}$. A hybrid precoder $\mathbf{P}_{t}=\mathbf{A}_{t} \mathbf{D}_{t}$ with dimensions $T_{x} \times N_{s}$ is applied at the transmitting side. The transmitted signal can be written as $\mathbf{x}=\mathbf{A}_{t} \mathbf{D}_{t} \mathbf{s}$, where $\mathbf{x} \in \mathbb{C}^{T_{x} \times 1}, \mathbf{s}$ is the symbol vector, $\mathbf{A}_{t}$ is the analog precoding matrix with dimensions $T_{x} \times N_{t}$, and $\mathbf{D}_{t} \in \mathbb{C}^{N_{t} \times N_{s}}$ is the BB digital precoding matrix. Similarly, at the receiver, a hybrid postcoding operation is performed $\mathbf{P}_{r}=\mathbf{A}_{r} \mathbf{D}_{r}$, with matrix $\mathbf{A}_{r}$ having dimensions $R_{x} \times N_{r}$ and $\mathbf{D}_{r} \in \mathbb{C}^{N_{r} \times N_{s}}$. Without loss of generality, we assume the entries of the data vector $s$ are zero mean independent variables with power $\sigma_{s}^{2}$.

We now explore the system and design constraints on the precoding matrices. Firstly, exploiting the unit power on each user streams, the constraint of $\left\|\mathbf{A}_{t} \mathbf{D}_{t}\right\|_{F}^{2}=N_{s}$ is imposed on the precoder design to account for the limited transmission power budget. The function of analog precoding and postcoding matrices $\mathbf{A}_{t}$ and $\mathbf{A}_{R}$ is to map the signals from the RF chains to the transmitting/receiving antennas.
These matrices implement the analog phase-shifting networks for the ease of design and implementation in the RF domain. Since $\mathbf{A}_{t}$ contain phase-shifters, it is clear that they satisfy the unit-modulus constraint. Further, the focus of this work is on the low-complexity partially-connected architecture, this further imposes structural constraints on the analog precoding/postcoding matrix as,

$$
\mathbf{A}_{t}=\left[\begin{array}{cccc}
\mathbf{a}_{1} & \mathbf{0} & \ldots & \mathbf{0} \\
\mathbf{0} & \mathbf{a}_{2} & \ldots & \mathbf{0} \\
\vdots & & \ddots & \mathbf{0} \\
\mathbf{0} & \mathbf{0} & \ldots & \mathbf{a}_{N_{t}}
\end{array}\right]
$$

where each $\mathbf{a}_{i}$ is $\frac{T_{x}}{N_{t}} \times 1$ dimensional vector consisting of unit-modulus entries ${ }^{1}$. This structure indicates that each RF chain is connected to only a subset of transmitting antennas having $\frac{T_{x}}{N_{t}}$ antennas in each subset. A similar structure for the receiver's analog precoding matrix can also be written with each sub-vector having dimensions $\frac{R_{x}}{N_{r}} \times 1$.

We consider a narrow-band block-fading propagation channel, thus the received signal before and after the postcoding operation is,

$$
\begin{aligned}
\mathbf{y} & =\mathbf{H P}_{t} \mathbf{s}+\mathbf{n}, \\
\mathbf{y}_{r} & =\mathbf{P}_{r}^{H} \mathbf{y},
\end{aligned}
$$

respectively, where $\mathbf{H} \in \mathbb{C}^{R_{x} \times T_{x}}$ is the channel matrix, and $\mathbf{n} \in \mathbb{C}^{R_{x} \times 1}$ is independent identically distributed (i.i.d) circular-symmetric complex Gaussian noise, $\mathcal{C N}\left(\mathbf{0}, \sigma_{n}^{2} \mathbf{I}\right), \sigma_{n}^{2}$ represents the noise variance. Considering the symbol vector to be zero-mean, complex circular-symmetric complex Gaussian with i.i.d entries, then its second order statistical characterization is, $\mathbb{E}\left\{\mathbf{s s}^{H}\right\}=\sigma_{s}^{2} \mathbf{I}$, where $\mathbb{E}\{\cdot\}$ is the expectation operator and $\sigma_{s}^{2}$ is the variance of $s_{i}, \forall i$. The spectral efficiency (SE) of such a system as given in [3], [5] is,

$$
\mathrm{SE}=\log _{2} \operatorname{det}\left(\mathbf{I}+\mathbf{R}_{n}^{-1} \mathbf{P}_{r}^{H} \mathbf{H} \mathbf{P}_{t} \mathbf{P}_{t}^{H} \mathbf{H}^{H} \mathbf{P}_{r}\right),
$$

where $\mathbf{R}_{n}=\sigma_{n}^{2} \mathbf{P}_{r}^{H} \mathbf{P}_{r}$ and $\sigma_{s}^{2}=1$.

\section{B. Channel Model}

We consider a narrow-band Saleh-Valenzuela model to accurately capture the characteristics of a typical mmWave channel including severe path loss, limited scattering, the high correlation among antennas due to closely packed antenna arrays, etc., [24], [25]. Assuming $N_{p}$ propagation paths between the transmitter and the receiver, we have,

$$
\mathbf{H}=\sqrt{\frac{T_{x} R_{x}}{N_{p}}} \sum_{k=1}^{N_{p}} \alpha_{k} \mathbf{b}_{r}\left(\phi_{k}^{r}\right) \mathbf{b}_{t}\left(\phi_{k}^{t}\right)^{H},
$$

where $\mathbf{b}_{r}\left(\phi_{k}^{r}\right)$ and $\mathbf{b}_{t}\left(\phi_{k}^{t}\right)$ represents the normalized array response or steering vectors associated with receive and transmit antenna arrays respectively, $\alpha_{k} \in \mathbb{C}$, is the gain along the $k$-th path, $\phi_{k}^{r}$ and $\phi_{k}^{t}$ are the azimuth angle of arrival and departure, respectively for the $k$-th path. A uniform linear array (ULA) of

\footnotetext{
${ }^{1}$ Without loss of generality we assume $T_{x}$ to be a multiple of $N_{t}$.
} 
isotropic antenna elements is considered at the transmitter and at the receiver. The associated steering vector in a direction $\phi$ is written as,

$$
\mathbf{b}(\phi)=\sqrt{\frac{1}{M}}\left[1, e^{j k d \sin (\phi)}, \ldots, e^{j(M-1) k d \sin (\phi)}\right]
$$

where $k=\frac{2 \pi}{\lambda}$ is the wavenumber, $\lambda$ is the carrier wavelength, $M$ is the number of antenna elements in the array and $d$ is the inter-element spacing between two antenna elements.

\section{Problem Formulation}

The objective is to maximize the SE of the system subject to discussed constraints to find the optimal hybrid precoders and postcoders. This can be formulated as,

$$
\begin{array}{cl}
\mathcal{P}_{1}: \max _{\mathbf{A}_{t}, \mathbf{D}_{t}, \mathbf{A}_{r}, \mathbf{D}_{r}} & \operatorname{SE}\left(\mathbf{A}_{t}, \mathbf{D}_{t}, \mathbf{A}_{r}, \mathbf{D}_{r}\right) \\
\text { subject to } \quad & \mathbf{A}_{t} \in \chi_{t}, \mathbf{A}_{r} \in \chi_{r} \\
& \left\|\mathbf{A}_{t} \mathbf{D}_{t}\right\|_{F}^{2}=N_{s},
\end{array}
$$

where $\chi_{t}$ and $\chi_{r}$ are the sets imposing structural constraints on the design of analog precoder and postcoder, respectively. Problem $\mathcal{P}_{1}$ is nonconvex and the joint design of precoders and postcoders becomes intractable. In fact, the SE maximization problem is intractable even for the fully-digital case. Therefore, instead of solving the SE maximization problem, it is proposed in the literature to decouple the transmitterreceiver problem. Then, hybrid precoder may be designed by maximizing the mutual information (MI) [3]. The MI maximization problem is given by,

$$
\begin{aligned}
& \mathcal{P}_{2}: \max _{\mathbf{A}_{t}, \mathbf{D}_{t}} \log _{2} \operatorname{det}\left(\mathbf{I}+\sigma_{n}^{-2} \mathbf{H} \mathbf{A}_{t} \mathbf{D}_{t} \mathbf{D}_{t}^{H} \mathbf{A}_{t}^{H} \mathbf{H}^{H}\right) \\
& \text { subject to } \mathbf{A}_{t} \in \chi_{t} \\
& \left\|\mathbf{A}_{t} \mathbf{D}_{t}\right\|_{F}^{2}=N_{s} \text {. }
\end{aligned}
$$

Still, problem $\mathcal{P}_{2}$ is nonconvex and difficult to optimize. As mentioned earlier, the MI maximization problem can be equivalently formulated as the Frobenius norm approximation between the fully-digital and the product of A/D preocders as,

$$
\begin{gathered}
\mathcal{P}_{3}: \min _{\substack{\mathbf{A}_{t}, \mathbf{D}_{t} \\
\text { subject to }}}\left\|\mathbf{P}_{t} \in \mathbf{A}_{t} \mathbf{D}_{t}\right\|_{F}^{2} \\
\left\|\mathbf{A}_{t} \mathbf{D}_{t}\right\|_{F}^{2}=N_{s} .
\end{gathered}
$$

The unconstrained fully-digital precoder $\mathbf{P}_{t}$ for a single user MIMO system is obtained from the singular value decomposition (SVD) of the channel matrix and the water-filling algorithm [26]. On the other hand, a minimum mean square error (MMSE) criterion based receiver is employed for the design of postocoders [3] as,

$$
\begin{gathered}
\mathcal{P}_{4}: \min _{\substack{\mathbf{A}_{r}, \mathbf{D}_{r} \\
\text { subject to } \mathbf{A}_{r} \in \chi_{r} .}} \mathbb{E}\left\|\mathbf{s}-\mathbf{D}_{r}^{H} \mathbf{A}_{r}^{H} \mathbf{y}\right\|_{2}^{2} \\
\text { subje }
\end{gathered}
$$

Problem $\mathcal{P}_{4}$ is also nonconvex due to the product of $\mathrm{A} / \mathrm{D}$ postcoders and the unit-modulus constraint on the analog postcoding matrix. An equivalent reformulation of the above problem is found in [3] as,

$$
\begin{aligned}
\mathcal{P}_{5}: & \min _{\substack{\mathbf{A}_{r}, \mathbf{D}_{r} \\
\text { subject to } \mathbf{A}_{r} \in \chi_{r}}}\left\|\mathbb{E}\left\{\mathbf{y} \mathbf{y}^{H}\right\}^{\frac{1}{2}}\left(\mathbf{P}_{r}-\mathbf{A}_{r} \mathbf{D}_{r}\right)\right\|_{F}^{2} \\
&
\end{aligned}
$$

where $\mathbb{E}\left\{\mathbf{y} \mathbf{y}^{H}\right\}^{\frac{1}{2}}$ is the positive square root of the covariance matrix of the received signal before postocoding $\mathbb{E}\left\{\mathbf{y} \mathbf{y}^{H}\right\}$. Problems $\mathcal{P}_{3}$ and $\mathcal{P}_{5}$ have a similar mathematical structure. Thus, we focus on the precoder design and utilize the derived algorithms for the receiver optimization. For brevity and notational simplicity from now onward we drop the subscripts $(\cdot)_{t}$ and $(\cdot)_{r}$, and consider the general CMCMF problem.

The CMCMF problem is written as,

$$
\begin{gathered}
\mathcal{P}_{6}: \min _{\mathbf{A}, \mathbf{D}}\|\mathbf{P}-\mathbf{A D}\|_{F}^{2} \\
\text { subject to } \mathbf{A} \in \mathcal{A} \\
\|\mathbf{A D}\|_{F}^{2}=N_{s},
\end{gathered}
$$

where $\mathbf{P}, \mathbf{A}$, and $\mathbf{D}$ denotes the fully digital, analog, and $\mathrm{BB}$ digital precoding matrices. The set $\mathcal{A}$ introduces unitmodulus as well as structural constraints on the design of analog precoding matrix as shown in (1).

\section{HYBRID PRE/Postcoding FOR THE PARTIALly Connected Architecture}

In the partially-connected architecture each RF chain is connected to $\frac{T_{x}}{N_{t}}$ number of antennas at the transmitter side. The constraint set $\mathcal{A}$ of problem $\mathcal{P}_{6}$ follows the structure of the matrix shown in (1), such that $\left\{\left|\mathbf{a}_{i}(j)\right|^{2}=1,1 \leq\right.$ $\left.i \leq N_{t}, 1 \leq j \leq T_{x} / N_{t}\right\}$, where $\mathbf{a}_{i}(j)$ denotes the $j$-th entry of the $i$-th sub-vector $\mathbf{a}_{i}$. As will be discussed later, to alleviate the differentiability issues with the modulus function, here we have constructed the set using squared unit-modulus constraint. This change does not affect the optimality of solution to the problem.

The structural constraint on the analog precoding matrix significantly simplifies the power constraint of problem $\mathcal{P}_{6}$ as, $\|\mathbf{A D}\|_{F}^{2}=\|\mathbf{D}\|_{F}^{2}=\frac{N_{s} N_{t}}{T_{x}}$, the last equality follows from the orthogonality among the columns of matrix $\mathbf{A}$, that is $\mathbf{A}^{H} \mathbf{A}=$ $\frac{T_{x}}{N_{t}} \mathbf{I}$. Therefore, problem $\mathcal{P}_{6}$ is reformulated as,

$$
\begin{aligned}
& \mathcal{P}_{7}: \min _{\mathbf{A}, \mathbf{D}}\|\mathbf{P}-\mathbf{A D}\|_{F}^{2} \\
& \text { subject to } \mathbf{A} \in \mathcal{A}, \\
&\|\mathbf{D}\|_{F}^{2}=\frac{N_{s} N_{t}}{T_{x}}=\beta .
\end{aligned}
$$

The above problem is nonconvex since neither the objective nor the constraints are convex.

\section{A. Proposed Solution}

The objective function of problem $\mathcal{P}_{7}$ is partially convex in one variable given the other variable. We exploit this partial convexity and first adopt the variable elimination approach. 
Considering the matrix $\mathbf{A}$ to be given, concentrating the problem with respect to the matrix $\mathbf{D}$ as,

$$
\begin{gathered}
\mathcal{P}_{8}: \quad \min _{\mathbf{D}}\|\mathbf{P}-\mathbf{A D}\|_{F}^{2} \\
\text { subject to }\|\mathbf{D}\|_{F}^{2}=\beta .
\end{gathered}
$$

Expanding the objective function, we get,

$$
\begin{gathered}
\mathcal{P}_{9}: \min _{\mathbf{D}} \operatorname{Tr}\left(\mathbf{P}^{H} \mathbf{P}-2 \operatorname{Re}\left(\mathbf{P}^{H} \mathbf{A D}\right)+\mathbf{D}^{H} \mathbf{A}^{H} \mathbf{A D}\right) \\
\text { subject to }\|\mathbf{D}\|_{F}^{2}=\beta .
\end{gathered}
$$

Once again using the orthogonality of matrix $\mathbf{A}$, we get the following problem,

$$
\mathcal{P}_{10}: \min _{\mathbf{D}} \operatorname{Tr}\left(\mathbf{P}^{H} \mathbf{P}-2 \operatorname{Re}\left(\mathbf{P}^{H} \mathbf{A D}\right)+\frac{T_{x}}{N_{t}} \mathbf{D}^{H} \mathbf{D}\right)
$$$$
\text { subject to }\|\mathbf{D}\|_{F}^{2}=\beta \text {. }
$$

After ignoring the constant terms and using the constraint in the objective function, we end up with the following formulation,

$$
\begin{gathered}
\mathcal{P}_{11}: \min _{\mathbf{D}}-\operatorname{Tr}\left(\operatorname{Re}\left(\mathbf{P}^{H} \mathbf{A D}\right)\right) \\
\text { subject to }\|\mathbf{D}\|_{F}^{2}=\beta .
\end{gathered}
$$

Problem $\mathcal{P}_{11}$ is reformulated as,

$$
\begin{gathered}
\mathcal{P}_{12}: \min _{\mathbf{D}}\left\|\mathbf{A}^{H} \mathbf{P}-\mathbf{D}\right\|_{F}^{2} \\
\text { subject to }\|\mathbf{D}\|_{F}^{2}=\beta .
\end{gathered}
$$

It is readily seen that the above problem admits the following closed-form solution,

$$
\mathbf{D}=\beta \frac{\mathbf{A}^{H} \mathbf{P}}{\left\|\mathbf{A}^{H} \mathbf{P}\right\|_{F}} .
$$

On substituting this solution back into the objective function of problem $\mathcal{P}_{7}$, we get,

$$
\begin{gathered}
\mathcal{P}_{13}: \min _{\mathbf{A}}\left\|\mathbf{P}-\beta \mathbf{A} \frac{\mathbf{A}^{H} \mathbf{P}}{\left\|\mathbf{A}^{H} \mathbf{P}\right\|_{F}}\right\|_{F}^{2} \\
\text { subject to } \mathbf{A} \in \mathcal{A} .
\end{gathered}
$$

Expanding the objective function in problem $\mathcal{P}_{13}$,

$$
\begin{gathered}
\operatorname{Tr}\left\{\mathbf{P}^{H} \mathbf{P}-2 \beta \operatorname{Re}\left(\mathbf{P}^{H} \mathbf{A} \frac{\mathbf{A}^{H} \mathbf{P}}{\left\|\mathbf{A}^{H} \mathbf{P}\right\|_{F}}\right)\right\} \\
+\beta^{2} \operatorname{Tr}\left\{\frac{\mathbf{P}^{H} \mathbf{A}}{\left\|\mathbf{A}^{H} \mathbf{P}\right\|_{F}} \mathbf{A}^{H} \mathbf{A} \frac{\mathbf{A}^{H} \mathbf{P}}{\left\|\mathbf{A}^{H} \mathbf{P}\right\|_{F}}\right\} .
\end{gathered}
$$

The above function is further simplified and we obtain the following minimization problem,

$$
\begin{gathered}
\mathcal{P}_{14}: \min _{\mathbf{A}}-\left\|\mathbf{A}^{H} \mathbf{P}\right\|_{F} \\
\text { subject to } \mathbf{A} \in \mathcal{A} .
\end{gathered}
$$

Equivalently, the above problem can be reformulated as the following maximization problem,

$$
\begin{gathered}
\mathcal{P}_{15}: \max _{\mathbf{A}}\left\|\mathbf{A}^{H} \mathbf{P}\right\|_{F}^{2} \\
\text { subject to } \mathbf{A} \in \mathcal{A} .
\end{gathered}
$$

Problem $\mathcal{P}_{15}$ is nonconvex because of the constraint set. In order to solve this problem, we now adopt the MM framework. The first step of this approach is to construct a tight minorizer of the objective function around a feasible initialpoint, followed by its iterative maximization. Let us denote the objective function of problem $\mathcal{P}_{15}$ as,

$$
g(\mathbf{A})=\sum_{i=1}^{N_{t}} \tilde{\mathbf{a}}_{i}^{H} \mathbf{P} \mathbf{P}^{H} \tilde{\mathbf{a}}_{i}
$$

where $\tilde{\mathbf{a}}_{i}$ is the $i$-th column of the matrix $\mathbf{A}$. Clearly, $g(\mathbf{A})$ is the summation of $N_{t}$ convex functions and it is a well known fact that a convex function is lower bounded by its first-order Taylor's expansion [27]. Using this the minorizing function of the $i$-th component of the objective function is given by,

$$
\begin{aligned}
\tilde{\mathbf{a}}_{i}^{H} \mathbf{P} \mathbf{P}^{H} \tilde{\mathbf{a}}_{i} \geq \tilde{\mathbf{a}}_{i, k}^{H} \mathbf{P} \mathbf{P}^{H} \tilde{\mathbf{a}}_{i, k} \\
+2 \operatorname{Re}\left(\left(\tilde{\mathbf{a}}_{i}-\tilde{\mathbf{a}}_{i, k}\right)^{H} \mathbf{P} \mathbf{P}^{H} \tilde{\mathbf{a}}_{i, k}\right) .
\end{aligned}
$$

In (22), $\tilde{\mathbf{a}}_{i, k}$ represents the solution to $\tilde{\mathbf{a}}_{i}$ available at $k$-th iteration. The overall objective function can be minorized as,

$$
\begin{aligned}
g(\mathbf{A}) \geq \tilde{g}\left(\mathbf{A} ; \mathbf{A}_{k}\right) & =\sum_{i=1}^{N_{t}} \tilde{\mathbf{a}}_{i, k}^{H} \mathbf{P} \mathbf{P}^{H} \tilde{\mathbf{a}}_{i, k} \\
& +2 \operatorname{Re}\left(\left(\tilde{\mathbf{a}}_{i}-\tilde{\mathbf{a}}_{i, k}\right)^{H} \mathbf{P} \mathbf{P}^{H} \tilde{\mathbf{a}}_{i, k}\right) .
\end{aligned}
$$

where $\mathbf{A}_{k}$ is matrix with $\tilde{\mathbf{a}}_{i, k}$, being the columns. Therefore, after neglecting the constant terms of the above surrogate function, the minorized version of the problem $\mathcal{P}_{15}$ can be written as,

$$
\begin{gathered}
\mathcal{P}_{16}: \max _{\mathbf{A}} \sum_{i=1}^{N_{t}} \operatorname{Re}\left(\tilde{\mathbf{a}}_{i}^{H} \mathbf{P} \mathbf{P}^{H} \tilde{\mathbf{a}}_{i, k}\right) \\
\text { subject to } \mathbf{A} \in \mathcal{A} .
\end{gathered}
$$

Problem $\mathcal{P}_{16}$ can be further reformulated to,

$$
\begin{gathered}
\mathcal{P}_{17}: \min _{\mathbf{A}} \sum_{i=1}^{N_{t}}\left\|\tilde{\mathbf{a}}_{i}-\mathbf{P} \mathbf{P}^{H} \tilde{\mathbf{a}}_{i, k}\right\|_{2}^{2} \\
\text { subject to } \mathbf{A} \in \mathcal{A} .
\end{gathered}
$$

At this point it is important to note that each column of the matrix $\mathbf{A}$ has only $T_{x} / N_{t}$ unit-modulus entries and rest of them are zeros. Using this fact we can rewrite the problem as,

$$
\begin{gathered}
\mathcal{P}_{18}: \min _{\mathbf{A}} \sum_{i=1}^{N_{t}}\left\|\mathbf{a}_{i}-\widetilde{\mathbf{P}}_{i} \widetilde{\mathbf{P}}_{i}^{H} \mathbf{a}_{i, k}\right\|_{2}^{2} \\
\text { subject to } \mathbf{A} \in \mathcal{C},
\end{gathered}
$$

where $\mathbf{a}_{i}$ is the $i$-th non-zero block of matrix $\mathbf{A}$ as shown in (1) and $\mathbf{a}_{i, k}$ is its corresponding $k$-th iterate. The matrix $\widetilde{\mathbf{P}}_{i}=\mathbf{P}_{(i-1) \frac{T x}{N_{t}}+1: i \frac{T_{x}}{N_{t}},: ;}, \forall \in\left[1, N_{t}\right]$, is the $i$-th sub-matrix formed by extracting the $\left[(i-1) \frac{T x}{N_{t}}+1\right]$-th to $i \frac{T_{x}}{N_{t}}$-th rows of matrix $\mathbf{P}$. It is easy to see that problem $\mathcal{P}_{18}$ admits the following closed-form solution,

$$
\mathbf{a}_{i}=e^{j \arg \left(\widetilde{\mathbf{P}} \widetilde{\mathbf{P}}^{H} \mathbf{a}_{i, k}\right)}, \forall i=1,2, \ldots, N_{t} .
$$




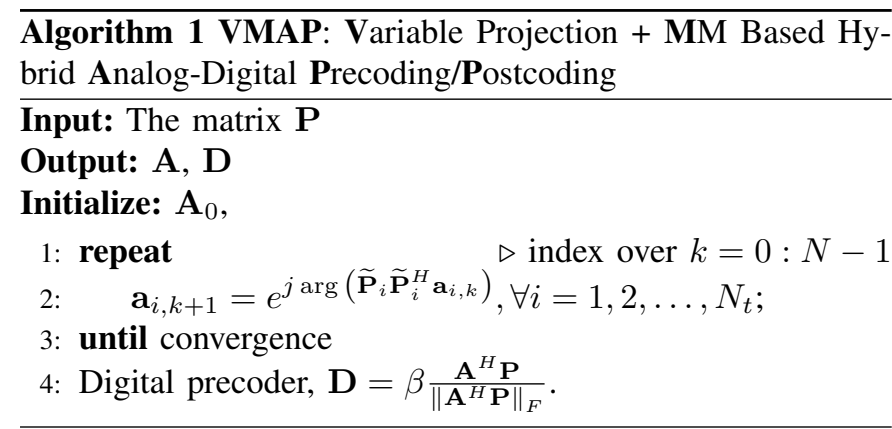

It is important to note that, as the objective function and the constraint set is separable in $\mathbf{a}_{i}$ 's, the problem can also be solved in a parallel manner, which is readily seen from the above solution. Once, the solution sequence of the analog precoding matrix $\left\{\mathbf{A}_{k}\right\}$ converges, the digital precoder is obtained using the closed-form solution from (18). The steps of the algorithm is summarized in Algorithm 1, namely VMAP (Variable Projection + MM Based Hybrid Analog-Digital Precoding/Postcoding). The convergence to a stationary point is stated in Theorem III.1.

Theorem III.1. Let $\left\{\mathbf{A}_{k}\right\}$ be the sequence of iterates generated by Algorithm VMAP. Then, every limit point of the sequence $\left\{\mathbf{A}_{k}\right\}$ is a stationary point of the problem $\mathcal{P}_{15}$.

Proof. For brevity the proof of convergence to a stationary point is omitted and will be available in a future journal submission.

Note that in order to compute SE of the system, we use the same algorithm for hybrid postcoder design by appropriately changing the dimensions of matrices $\mathbf{A}$ and $\mathbf{D}$.

\section{B. Complexity Analysis of VMAP}

Algorithm VMAP requires a matrix-matrix, matrix-vector multiplications and a phase computation operation at each iteration. As compared to SDR-ALTMIN proposed in [12], it does not require to solve the problem using alternating minimization. Matrix multiplication of the term $\widetilde{\mathbf{P}}_{i} \widetilde{\mathbf{P}}_{i}^{H}$ requires $O\left(\left(T_{x} / N_{t}\right)^{2} N_{s}\right)$ operations. The exponential operation can be carried in $O\left(\left(T_{x} / N_{t}\right) N_{s}\right)$ operations. Thus, our algorithm is computationally efficient and easily scalable to large dimensions.

\section{Simulation Results}

In this section, we numerically evaluate the performance of the VMAP algorithm and compare the SE achieved with that of the SDR-ALTMIN algorithm [12]. We consider a scenario with $T_{x}=144$ and $R_{x}=36$. The channel parameters are $N_{c}=5$ clusters and $N_{p}=10$ propagation paths. The element spacing of the ULA is set to $d=\frac{\lambda}{2}$.

The evolution of the objective function of problem $\mathcal{P}_{15}$ with number of iterations is shown in Figure 1. As expected, the objective function value increases monotonically with the number of iterations. Figure 2 shows the $\mathrm{SE}$ achieved by the algorithm at different signal-to-noise ratio (SNR) values.

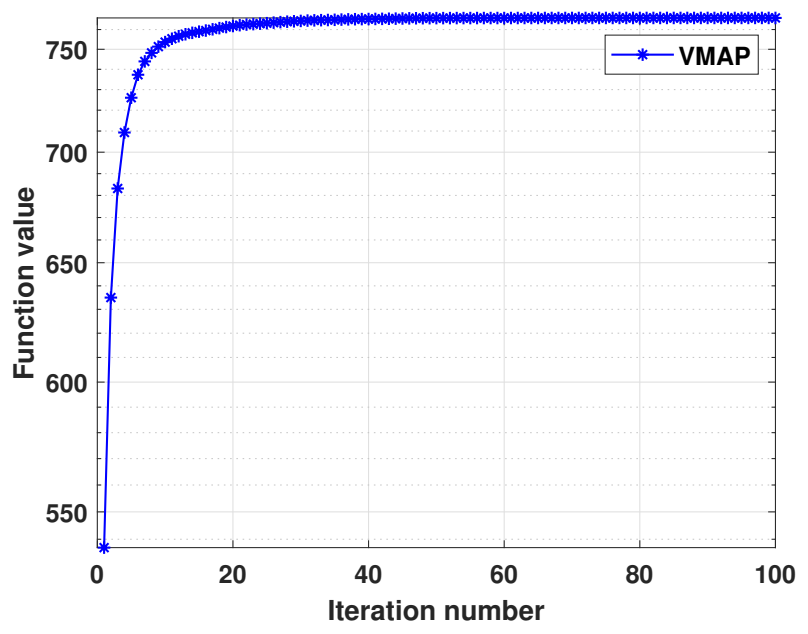

Fig. 1. Objective function variation with the number of iterations for the partially connected architecture, $N_{t}=N_{r}=N_{s}=6$.

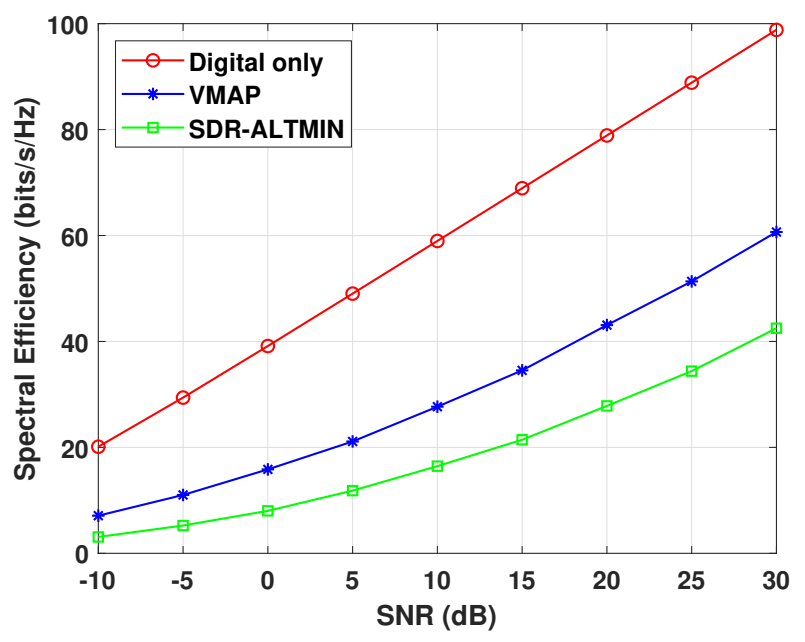

Fig. 2. Spectral Efficiency comparison for different algorithm for the partially connected architecture, when $N_{t}=N_{r}=N_{s}=6$.

Clearly visible, our algorithm achieves significant gains in SE compared to SDR-ALTMIN algorithm.

Figure 3 shows the variation of SE with the increasing number of RF chains and keeping the number of streams, transmitting, and receiving antennas fixed. The system dimensions used in this case are $T_{x}=144$ and $R_{x}=72$. For this plot, we have used different system dimensions to avoid divisibility issues with the factor $T_{x} / N_{t}$.

\section{CONCLUSions}

We have presented a low-complexity algorithm to design a hybrid transceiver based on partially-connected architecture for the single user point-to-point mmWave MIMO system. The proposed algorithm results in a closed-form expression for the digital precoder while using a simple iteration for the analog precoder. Further, the iterations converge to a stationary point. In comparison to the existing solutions, the proposed algorithm 


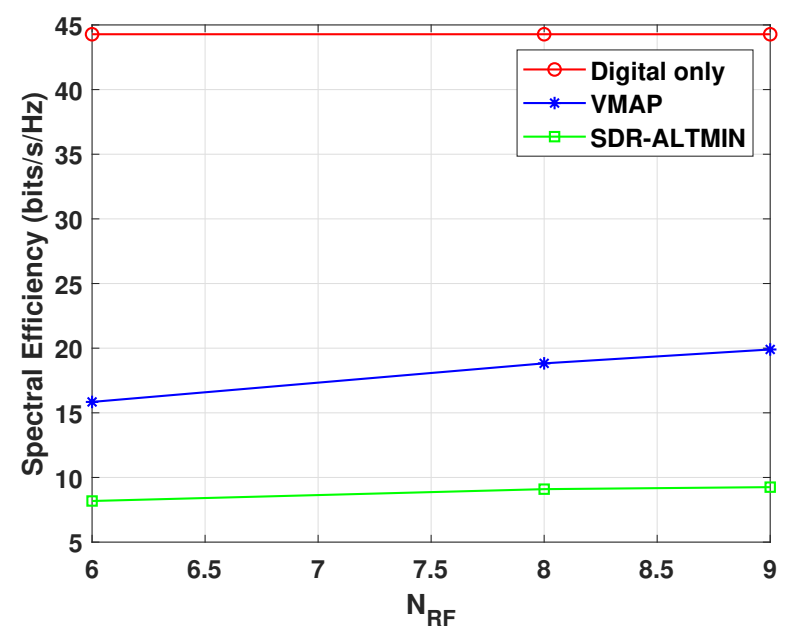

Fig. 3. Spectral Efficiency comparison for different algorithm for the partially connected architecture, when $N_{s}=6, N_{t}=N_{r}=N_{\mathrm{RF}}$, and SNR $=0 \mathrm{~dB}$.

does not require alternate minimization and toolboxes to solve the problem, making it more computationally efficient. Simulation results demonstrated notable improvement in SE achieved by the proposed method.

\section{REFERENCES}

[1] X. Zhang, A. F. Molisch, and S. Y. Kung, "Variable-phase-shift-based RF-baseband codesign for MIMO antenna selection," IEEE Trans. Signal Process., vol. 53, no. 11, pp. 4091-4103, Nov 2005.

[2] A. Alkhateeb, O. El Ayach, G. Leus, and R. W. Heath, "Channel estimation and hybrid precoding for millimeter wave cellular systems," IEEE J. Sel. Topics Signal Process., vol. 8, no. 5, pp. 831-846, Oct 2014.

[3] O. E. Ayach, S. Rajagopal, S. Abu-Surra, Z. Pi, and R. W. Heath, "Spatially sparse precoding in millimeter wave MIMO systems," IEEE Trans. Wireless Commun., vol. 13, no. 3, pp. 1499-1513, March 2014.

[4] M. R. Akdeniz, Y. Liu, M. K. Samimi, S. Sun, S. Rangan, T. S. Rappaport, and E. Erkip, "Millimeter wave channel modeling and cellular capacity evaluation," vol. 32, no. 6, pp. 1164-1179, June 2014.

[5] C. G. Tsinos, S. Maleki, S. Chatzinotas, and B. Ottersten, "On the energy-efficiency of hybrid analog-digital transceivers for single- and multi-carrier large Antenna array systems," IEEE J. Sel. Areas Commun., vol. 35, no. 9, pp. 1980-1995, Sep. 2017.

[6] D. J. Love and R. W. Heath, "Equal gain transmission in multiple-input multiple-output wireless systems," IEEE Trans. Commun., vol. 51, no. 7, pp. 1102-1110, July 2003.

[7] S. Hur, T. Kim, D. J. Love, J. V. Krogmeier, T. A. Thomas, and A. Ghosh, "Millimeter wave beamforming for wireless backhaul and access in small cell networks," IEEE Trans. Commun., vol. 61, no. 10, pp. 4391-4403, October 2013.

[8] T. Baykas, C. Sum, Z. Lan, J. Wang, M. A. Rahman, H. Harada, and S. Kato, "IEEE 802.15.3c: The first IEEE wireless standard for data rates over $1 \mathrm{~Gb} / \mathrm{s}$," IEEE Commun. Mag., vol. 49, no. 7, pp. 114-121, July 2011.

[9] A. F. Molisch, M. Z. Win, , and J. H. Winters, "Capacity of MIMO systems with antenna selection," IEEE Trans. Wireless Commun., vol. 4, no. 4, pp. 1759-1772, July 2005 .

[10] A. Gorokhov, D. A. Gore, and A. J. Paulraj, "Receive antenna selection for MIMO spatial multiplexing: Theory and algorithms," IEEE Trans. Signal Process., vol. 51, no. 11, pp. 2796-2807, Nov 2003.

[11] S. Sanayei and A. Nosratinia, "Antenna selection in MIMO systems," IEEE Commun. Mag., vol. 42, no. 10, pp. 68-73, Oct 2004.

[12] X. Yu, J. Shen, J. Zhang, and K. B. Letaief, "Alternating minimization algorithms for hybrid precoding in millimeter wave MIMO systems," IEEE J. Sel. Topics Signal Process., vol. 10, no. 3, pp. 485-500, April 2016.
[13] T. E. Bogale, L. B. Le, A. Haghighat, and L. Vandendorpe, "On the number of RF chains and phase shifters, and scheduling design with hybrid analog-digital beamforming," IEEE Trans. Wireless Commun., vol. 15, no. 5, pp. 3311-3326, May 2016.

[14] S. Payami, M. Ghoraishi, and M. Dianati, "Hybrid beamforming for large antenna arrays with phase shifter selection," IEEE Trans. Wireless Commun., vol. 15, no. 11, pp. 7258-7271, Nov 2016.

[15] S. Payami, M. Ghoraishi, M. Dianati, and M. Sellathurai, "Hybrid beamforming with a reduced number of phase shifters for massive MIMO systems," IEEE Trans. Veh. Technol., vol. 67, no. 6, pp. 48434851, June 2018 .

[16] A. Li and C. Masouros, "Hybrid analog-digital millimeter-wave MUMIMO transmission with virtual path selection," IEEE Commun. Lett., vol. 21, no. 2, pp. 438-441, Feb 2017.

[17] E. Vlachos, A. Kaushik, and J. Thompson, "Energy efficient transmitter with low resolution DACs for massive MIMO with partially connected hybrid architecture," in 2018 IEEE 87th Vehicular Technology Conference (VTC Spring), June 2018, pp. 1-5.

[18] M. Á. Vázquez, L. Blanco, and A. I. Pérez-Neira, "Hybrid analog-digital transmit beamforming for spectrum sharing backhaul networks," IEEE Trans. Signal Process., vol. 66, no. 9, pp. 2273-2285, May 2018.

[19] C. G. Tsinos, S. Chatzinotas, and B. Ottersten, "Hybrid analog-digital transceiver designs for mmwave amplify-and-forward relaying systems," in 2018 41st International Conference on Telecommunications and Signal Processing (TSP), July 2018, pp. 1-6.

[20] C. G. Tsinos, S. Maleki, S. Chatzinotas, and B. Ottersten, "On the energy-efficiency of hybrid analog-digital transceivers for large antenna array systems," in 2017 IEEE International Conference on Communications (ICC), May 2017, pp. 1-7.

[21] — , "Hybrid analog-digital transceiver designs for cognitive radio millimiter wave systems," in 2016 50th Asilomar Conference on Signals, Systems and Computers, Nov 2016, pp. 1785-1789.

[22] J. A. Tropp and A. C. Gilbert, "Signal recovery from random measurements via orthogonal matching pursuit," IEEE Trans. Inf. Theory, vol. 53, no. 12, pp. 4655-4666, Dec 2007.

[23] A. F. Molisch, V. V. Ratnam, S. Han, Z. Li, S. L. H. Nguyen, L. Li, and K. Haneda, "Hybrid beamforming for massive MIMO: A survey," IEEE Commun. Mag., vol. 55, no. 9, pp. 134-141, Sep. 2017.

[24] P. F. M. Smulders and L. M. Correia, "Characterisation of propagation in $60 \mathrm{GHz}$ radio channels," Electronics Communication Engineering Journal, vol. 9, no. 2, pp. 73-80, April 1997.

[25] H. Xu, V. Kukshya, and T. S. Rappaport, "Spatial and temporal characteristics of $60-\mathrm{GHz}$ indoor channels," IEEE J. Sel. Areas Commun., vol. 20, no. 3, pp. 620-630, April 2002.

[26] D. Tse and P. Viswanath, Fundamentals of Wireless Communication. Cambridge University Press, 2005.

[27] S. Boyd and L. Vandenberghe, Convex Optimization. Cambridge University Press, 2004. 